\title{
Selection of Salt-tolerant Yeast Strains Accumulating Glucosylceramide
}

\author{
Naoya TAKaKUwa ${ }^{1}$, Masao OHnishi ${ }^{2}$ and Yuji OdA ${ }^{1 *}$ \\ ${ }^{1}$ Department of Upland Agriculture, National Agricultural Research Center for Hokkaido Region, \\ Memuro, Kasai, Hokkaido 082-0071, Japan \\ ${ }^{2}$ Department of Agricultural and Life Science, Obihiro University of Agricultural and Veterinary Medicine, \\ Obihiro, Hokkaido 080-8555, Japan
}

Received January 31, 2005; Accepted March 22, 2005

\begin{abstract}
Debaryomyces hansenii NBRC 0855 accumulated high amounts of glucosylceramide relative to 25 strains of salt-tolerant yeasts in the genera Candida, Debaryomyces, Kodamaea, and Pichia. The constituents of glucosylceramide were principally 2-hydroxystearic acid as a fatty acid, 9-methyl-trans-4, trans-8sphingadienine as a sphingoid base and glucose as a hexose. Strain NBRC 0855 was able to produce glucosylceramide when cultured on actual effluent from a pickle manufacturer.
\end{abstract}

Keywords: Glucosylceramide, salt-tolerant yeast, Debaryomyces hansenii

Kimchi, a Korean fermented food product, has become popular in Japan over the last few years. It accounted for $30 \%$ of the total pickled food produced in 2002 and dominates the market for such foodstuffs in Japan. Kimchi is made by fermenting Chinese cabbage, radish and many other types of vegetables. First, these vegetables are trimmed, cut, and salted, before the various spices and ingredients are added (Cheigh and Park, 1994). The resulting brine, which contains $10-15 \% \mathrm{NaCl}$ and some sugars that are extracted from the vegetables, cannot be discharged into rivers without complicated treatment. Disposal of this brine causes serious problems for manufacturers given that volumes are estimated to be at least 200,000 tons annually.

In Wakayama Prefecture, Japan, water, salts, and citric acid in plum vinegar, a by-product of the pickled plum industry, are used as raw materials for foods and cosmetics. Choi and Park (1999) cultured the osmotolerant yeast, Pichia guilliermondii A9, for potential application as singlecell protein in the brine waste derived from kimchi production. We sought to determine whether the production of glucosylceramide from the cells of salt-tolerant yeast grown in waste brine was possible. Glucosylceramide is abundant in animal cerebra and nerve organs (Cavanna and Rapport, 1967) and is one of the ceramides used as an ingredient in cosmetics (Loden, 2003) and food supplements (Sugawara et al., 2003). Commercially available preparations of ceramide extracted from plants are principally composed of glucosylceramide (Sugawara and Miyazawa, 1999). The content of glucosylceramide is so limited (0.01-0.02\% as dry mass) that the costs associated with extraction and purification inflate the retail price of the final product considerably. Consequently, products

E-mail: yujioda@affrc.go.jp containing as little as $3 \%$ glucosylceramide retail at $200-$ 300 yen per gram. While members of the genera Saccharomyces, Torulaspora, Zygosaccharomyces, and Kluyveromyces, S. kluyveri, Z. cidri, Z. fermentati, K. lactis, K. thermotolerans, and $K$. waltii all synthesize glucosylceramide (Takakuwa et al. 2002), these strains cannot grow in the presence of $10 \% \mathrm{NaCl}$. We therefore selected a strain of salt-tolerant yeast that can propagate in waste brine to produce glucosylceramide.

All of the strains used in this study (Table 1) were isolated from "shoyu" mash, fermented foods, or alcoholic beverages, and were obtained from the NITE Biological Resource Center (NBRC), Chiba, Japan.

Yeast cells were grown in $40 \mathrm{ml}$ of YNBD medium containing $1 \%$ glucose and $0.67 \%$ yeast nitrogen base without amino acids supplemented with $10.0 \% \mathrm{NaCl}$ in a 200 $\mathrm{ml}$ Erlenmeyer flask at $25^{\circ} \mathrm{C}$ for $72 \mathrm{~h}$ with shaking (180 rpm) unless otherwise stated.

The cultured cells were collected, lyophilized after being washed twice with distilled water and stored at $-20^{\circ} \mathrm{C}$. Glucosylceramides in the yeast cells were extracted and quantified as described previously (Takakuwa et al. 2005). The data reported here are the average values with their associated standard deviations obtained from three independent experiments.

All 26 strains of salt-tolerant yeasts grew to some extent in YNBD medium containing 10\% $\mathrm{NaCl}$ and glucosylceramide was detected in 22 strains (Table 1). After considering the yield in biomass, Debaryomyces hansenii NBRC 0855 appeared to be the most suitable strain for the production of glucosylceramide from waste containing a high concentration of salt.

The effect of $\mathrm{NaCl}$ on growth and glucosylceramide content was followed in strain NBRC 0855 (Fig. 1). The absence of $\mathrm{NaCl}$ stimulated growth and resulted in doubl- 
ing the biomass while reducing the glucosylceramide content to approximately one-third.

Strain NBRC 0855 was cultured in waste brine, actual effluent from a pickle manufacturer, containing $10 \% \mathrm{NaCl}$ and $3.0 \%$ glucose. The biomass yield and glucosylceramide content were $4.76(\mathrm{mg} / \mathrm{ml})$ and $0.11(\mathrm{mg} /[\mathrm{g}$ cells $])$, respectively. Components of glucosylceramide recovered using thin layer chromatography were analyzed by gas chromatography-mass spectrometry according to previously described methods (Takakuwa et al., 2002). Table 2 compares fatty acids and sphingoid bases of glucosylceramide from strain NBRC 0855 from three media. The sugar moiety included was a single molecule of glucose in any of the glucosylceramides. The fatty acid was exclusively 2-hydroxystearic acid in all of the cells. The principal sphingoid base of glucosylceramide from the cells grown in YNBD media with and without $\mathrm{NaCl}$ was 9-methyltrans-4, trans-8-sphingadienine, as found in other fungi and yeasts (Takakuwa et al., 2002). However, the cells grown in waste brine included trans-4, trans-8-sphingadienine instead of 9-methyl-trans-4, trans-8-sphingadienine. It is still unknown whether some compounds in waste brine inhibited the methyl transfer or whether those compounds essential for methyl transfer were lacking in the waste brine. The presence of the methyl unit may not affect physiological activity because the daily ingestion of glucosylceramides from both plants and fungi has been shown to suppress colon carcinoma in humans (Aida et al., 2004; Aida et al., 2005).

In conclusion, $D$. hansenii NBRC 0855 may be used for the production of a valuable product, glucosylceramide, from residual sugars in waste brine.

Acknowledgments This work was partly supported by a Grantin-Aid from Bio-oriented Technology Research Advancement Institution of National Agricultural and Bio-oriented Research Organization.

Table 1. Glucosylceramide contents in strains of salt-tolerant yeasts.

\begin{tabular}{|c|c|c|c|}
\hline Species & NBRC & $\begin{array}{r}\text { Biomass }^{a} \\
(\mathrm{mg} / \mathrm{ml})\end{array}$ & $\begin{array}{c}\text { Glucosylceramide } \\
(\mathrm{mg} /[\mathrm{g} \text { cells }])\end{array}$ \\
\hline \multirow[t]{2}{*}{ Candida etchellsii } & 1592 & $0.92 \pm 0.04$ & $<0.1$ \\
\hline & 1942 & $0.74 \pm 0.06$ & $<0.1$ \\
\hline C. lactis-condensi & 10288 & $0.35 \pm 0.04$ & $<0.1$ \\
\hline \multirow[t]{2}{*}{ C. polymorpha } & 10662 & $1.57 \pm 0.02$ & $0.09 \pm 0.01$ \\
\hline & 10663 & $1.24 \pm 0.17$ & $0.05 \pm 0.01$ \\
\hline \multirow[t]{3}{*}{ C. versatilis } & 1941 & $0.48 \pm 0.02$ & $0.12 \pm 0.01$ \\
\hline & 10056 & $0.90 \pm 0.08$ & $0.11 \pm 0.02$ \\
\hline & 10664 & $0.37 \pm 0.01$ & $0.03 \pm 0.01$ \\
\hline Debaryomyces carsonii & 10572 & $1.40 \pm 0.01$ & $0.57 \pm 0.04$ \\
\hline D. etchellsii & 1987 & $0.98 \pm 0.02$ & $0.12 \pm 0.01$ \\
\hline \multirow[t]{5}{*}{ D. hansenii } & 0026 & $0.72 \pm 0.00$ & $0.64 \pm 0.11$ \\
\hline & 0093 & $0.72 \pm 0.12$ & $0.44 \pm 0.09$ \\
\hline & 0855 & $1.55 \pm 0.01$ & $0.75 \pm 0.05$ \\
\hline & 10142 & $0.70 \pm 0.04$ & $0.13 \pm 0.02$ \\
\hline & 10939 & $1.45 \pm 0.02$ & $0.46 \pm 0.07$ \\
\hline \multirow[t]{3}{*}{ D. hansenii var. fabri } & 0037 & $1.24 \pm 0.03$ & $0.43 \pm 0.07$ \\
\hline & 0058 & $0.67 \pm 0.01$ & $0.50 \pm 0.10$ \\
\hline & 0059 & $1.05 \pm 0.03$ & $0.49 \pm 0.06$ \\
\hline \multirow[t]{4}{*}{ D. hansenii var. hansenii } & 0032 & $0.76 \pm 0.04$ & $0.71 \pm 0.09$ \\
\hline & 0048 & $0.80 \pm 0.16$ & $0.42 \pm 0.03$ \\
\hline & 0066 & $0.67 \pm 0.03$ & $0.35 \pm 0.02$ \\
\hline & 0094 & $1.28 \pm 0.09$ & $0.24 \pm 0.05$ \\
\hline D. maramus & 10687 & $0.91 \pm 0.05$ & $0.72 \pm 0.07$ \\
\hline D. nepalensis & 0039 & $0.54 \pm 0.10$ & $<0.1$ \\
\hline Kodamaea ohmeri & 1271 & $2.02 \pm 0.04$ & $0.42 \pm 0.03$ \\
\hline Pichia subpelliculosa & 10666 & $3.36 \pm 0.18$ & $0.20 \pm 0.01$ \\
\hline
\end{tabular}

Each value is shown as the average \pm standard deviation. ${ }^{a}$ Cell weight as dry matter. 
Table 2. Composition of fatty acids and sphingoid bases in glucosylceramide from $D$. hansenii NBRC 0855 grown in three media.

\begin{tabular}{lccc}
\hline \multirow{2}{*}{ Component $^{a}$} & \multicolumn{3}{c}{ Media } \\
\cline { 2 - 4 } & YNBD & YNBD+10\% NaCl & Waste brine \\
\hline Fatty acid (\%) & 1 & 2 & 2 \\
16h:0 & 99 & 98 & 98 \\
18h:0 & & & \\
Sphingoid base (\%) & 15 & 8 & 4 \\
t 18:0 & 1 & 0 & 0 \\
t 20:0 & 1 & 3 & 10 \\
d18:0 & 2 & 1 & 3 \\
d18:1 $1^{4 t}$ & 22 & 12 & 74 \\
d18:2 & 59 & 76 & 9 \\
9-Me d18: $182^{4 t, 8 t}$ & 59 & & \\
\hline
\end{tabular}

${ }^{a}$ Abbreviations are as follows: 16h:0, 2-hydroxypalmitic acid; 18h:0, 2-hydroxystearic acid; t18:0, 4-hydroxysphinganine; t20:0, 4-hydroxyeicosasphinganine; d18:0, sphinganine; d18: $1^{4 t}$, trans-4-sphingenine; d18:2 $2^{4 t, 8 t}$, trans-4, trans-8-sphingadienine; and 9-Me d18:2 ${ }^{4 t, 8 t}$, 9-methyl-trans-4, trans-8-sphingadienine.
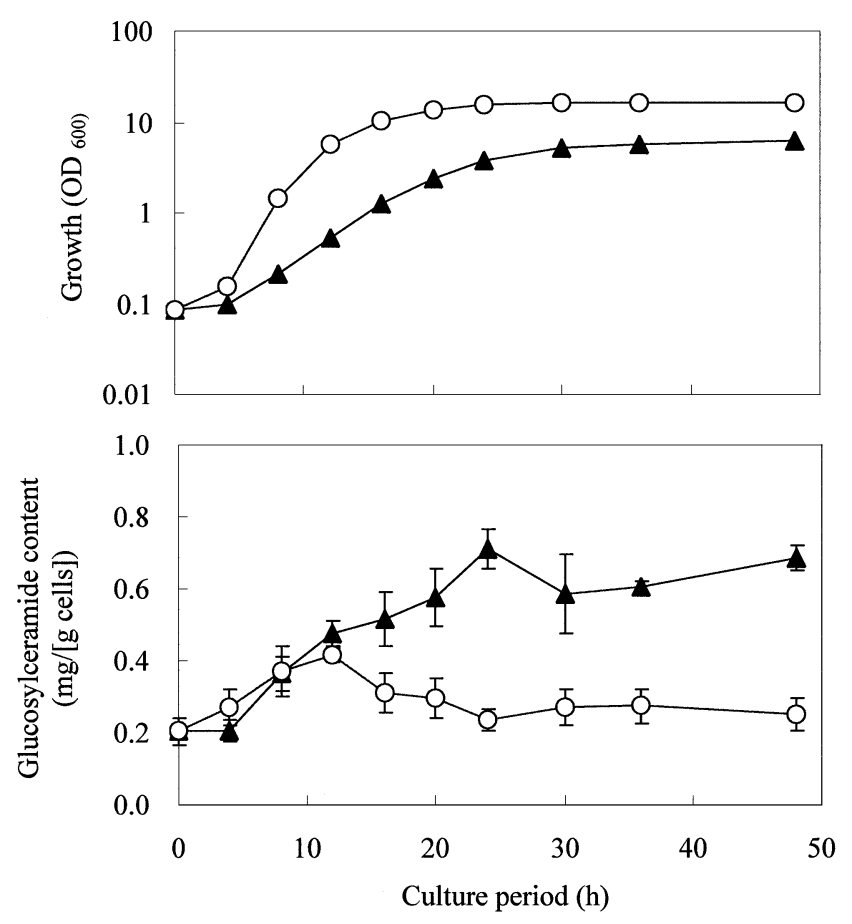

Fig. 1. Growth and glucosylceramide content of D. hansenii NBRC 0855 in medium with $(\mathbf{\Delta})$ and without $(\mathrm{O})$ added $10 \%$ $\mathrm{NaCl}$. Vertical bars indicate standard deviation.

\section{References}

Aida, K., Kinoshita, M., Sugawara, T., Ono, J., Miyazawa, T. and Ohnishi, M. (2004). Apoptosis inducement by plant and fungus sphingoid bases in human colon cancer cells. J. Oleo Sci., 53, 503-510.

Aida, K., Kinoshita, M., Sugawara, T., Tamura, M., Ono, J., Ueno, N. and Ohnishi, M. (2005). Prevention of aberrant crypt foci formation by dietary maize and yeasts in 1,2-dimethylhydrazinetreated mice. J. Oleo Sci., 54, 45-49.

Cavanna, R. and Rapport, M.M. (1967). An improved preparation of bovine brain proteolipid. J. Lipid Res., 8, 65-68.

Cheigh, H.K. and Park, K.Y. (1994). Biochemical, microbiological, and nutritional aspects of kimchi (Korean fermented vegetable products). Crit. Rev. Food Sci. Nutr., 34, 175-203.

Choi, M.H. and Park, Y.H. (1999). Growth of Pichia guilliermondii A9, and osmotolerant yeast, in waste brine generated from kimchi production. Bioresour. Technol., 70, 231-236.

Loden, M. (2003). Role of topical emollients and moisturizers in the treatment of dry skin barrier disorders. Am. J. Clin. Dermatol., 4, 771-788.

Sugawara, T., Kinoshita, M., Ohnishi, M., Nagata, J. and Saito, M. (2003). Digestion of maize sphingolipids in rats and uptake of sphingadienine by Caco-2 cells. J. Nutr., 133, 2777-2782.

Sugawara, T. and Miyazawa, T. (1999). Separation and determination of glycolipids from edible plant sources by highperformance liquid chromatography and evaporative lightscattering detection. Lipids, 34, 1231-1237.

Takakuwa, N., Saito, K., Ohnishi, M. and Oda, Y. (2005). Determination of glucosylceramide contents in crop tissues and byproducts from their processing. Bioresour. Technol., 96, 10891092.

Takakuwa, N., Kinoshita, M., Oda, Y. and Ohnishi, M. (2002). Existence of cerebroside in Saccharomyces kluyveri and its related species. FEMS Yeast Res., 2, 533-538. 